\title{
Upregulation of cell-surface mucin MUC15 in human nasal epithelial cells upon influenza A virus infection
}

Zhuang Gui Chen ${ }^{1,2+}$, Zhao Ni Wang ${ }^{1,2+}$, Yan Yan ${ }^{2,3}$, Jing Liu², Ting Ting He², Kim Thye Thong ${ }^{2}$, Yew Kwang Ong ${ }^{2}$, Vincent T. K. Chow ${ }^{4}$, Kai Sen Tan ${ }^{2 *}$ and De Yun Wang ${ }^{2}$

\begin{abstract}
Background: Cell-surface mucins are expressed in apical epithelial cells of the respiratory tract, and contribute a crucial part of the innate immune system. Despite anti-inflammatory or antiviral functions being revealed for certain cell-surface mucins such as MUC1, the roles of other mucins are still poorly understood, especially in viral infections.

Methods: To further identify mucins significant in influenza infection, we screened the expression of mucins in human nasal epithelial cells infected by H3N2 influenza A virus.

Results: We found that the expression of MUC15 was significantly upregulated upon infection, and specific only to active infection. While MUC15 did not interact with virus particles or reduce viral replication directly, positive correlations were observed between MUC15 and inflammatory factors in response to viral infection. Given that the upregulation of MUC15 was only triggered late into infection when immune factors (including cytokines, chemokines, EGFR and phosphorylated ERK) started to peak and plateau, MUC15 may potentially serve an immunomodulatory function later during influenza viral infection.
\end{abstract}

Conclusions: Our study revealed that MUC15 was one of the few cell-surface mucins induced during influenza infection. While MUC15 did not interact directly with influenza virus, we showed that its increase coincides with the peak of immune activation and thus MUC15 may serve an immunomodulatory role during influenza infection.

Keywords: MUC15, H3N2, Mucin, Immunomodulation

\section{Background}

Airway epithelium is the central component of the defense against respiratory pathogens through the combined functions of physical barrier and the regulation of both innate and adaptive immunity [1]. In the healthy state, integrated cell-cell junctions, the airway mucus layer and the beating cilia act as the physical barrier by clearance of particulates such as pathogens and preventing them from entry into submucosa. The airway mucus is a viscoelastic gel with a complicated composition including antimicrobial substances, cytokines and antioxidant proteins [2] where mucins also play the role of the structural framework.

\footnotetext{
* Correspondence: tan_ks@u.nus.edu

${ }^{\dagger}$ Zhuang Gui Chen and Zhao Ni Wang contributed equally to this work. ${ }^{2}$ Department of Otolaryngology, Yong Loo Lin School of Medicine, National University of Singapore, 1E Kent Ridge Road, Singapore 119228, Singapore Full list of author information is available at the end of the article
}

To date, 22 types of mucin proteins have been discovered in humans [3]. The mucins can be further divided into two groups according to their subcellular localization: secreted mucins and membrane-tethered mucins. Secreted mucins, such as MUC5AC and MUC5B, are large glycoproteins mainly produced by goblet cells and submucosal glands [4]. Conversely, membrane-tethered mucins, also known as cell-surface mucins, are encoded by MUC1, MUC3A/B, MUC4, MUC11, MUC12, MUC13, MUC15, MUC16, MUC17, MUC18, MUC20, MUC21 or $M U C 22$; and consist of transmembrane domains that anchor themselves to the plasma membrane, and some of them may shed their extracellular fragments into the airway tract cavity [3].

There are two distinctive mucus layers $[5,6]$. The apical layer is rich in the two well-known secreted mucins, MUC5AC and MUC5B; and this layer is stickier so that

(c) The Author(s). 2019 Open Access This article is distributed under the terms of the Creative Commons Attribution 4.0 International License (http://creativecommons.org/licenses/by/4.0/), which permits unrestricted use, distribution, and reproduction in any medium, provided you give appropriate credit to the original author(s) and the source, provide a link to the Creative Commons license, and indicate if changes were made. The Creative Commons Public Domain Dedication waiver (http://creativecommons.org/publicdomain/zero/1.0/) applies to the data made available in this article, unless otherwise stated. 
particulates in the airway tract cavity could cling to it and then be trapped. The lower layer, also called periciliary layer (PCL), is "watery" or less viscoelastic, and thus allows cilia to beat with less resistance. In this layer, most membrane-tethered mucins such as MUC1, MUC4 and MUC16 localize on microvilli, cilia or goblet cells. These tethered mucins are found to trap smaller adenoassociated virus. Therefore, unlike their secreted counterparts, cell surface mucins likely function as a selective barrier rather than a non-specific one [6,7].

Furthermore, cell-surface mucin may function as immunomodulatory factors during invasion of pathogens and allergens, working in concert with other components of the immune system to exert a suitable immune response. Among the cell surface mucins, MUC1 is the most studied cell-surface mucin and its anti-inflammatory role initiated by bacterial and viral infection has been well established. MUC1 is upregulated by respiratory virusinduced cytokines such as TNF $\alpha$ and IL8. However, MUC1 can then diminish the levels of these inflammatory factors by suppressing Toll-like receptor (TLR) pathway as a feedback loop [8-11]. Recently, it was reported that MUC1 defends against influenza virus by directly interacting with the viral particles and eliminating viral entry into respiratory epithelial cells [12]. These results implied that cell-surface mucins might regulate the immune response in airway epithelial cells during bacterial or viral infection so as to reduce inflammation's harmful effect on the host.

However, despite the many studies on MUC1, the role of other cell-surface mucins in the airway during viral infection has not been well illustrated and demonstrated. Considering that MUC1 plays a role in microbial infection, it is therefore interesting to investigate the expression of other cell-surface mucins in an airway infection model. We have previously established a human nasal epithelial cells (hNECs) model for influenza infection and the study of airway host factors [13-15]. Using this hNECs model, we investigated the expression and potential functions of these mucins in influenza A virus infection, which can potentially help identify other mucins that are significant in influenza infection as targets for diagnostic or treatment purposes.

\section{Methods}

\section{Cell culture}

This study was approved by the National Healthcare Group Domain-Specific Review Board of Singapore (Ethics approval number: DSRB D/11/228; IRB 13-509). Nasal epithelial biopsies were obtained from adult patients with inferior turbinate or nasal polyps, who were scheduled for septoplasty or polypectomy in the Department of Otolaryngology of the National University Hospital (Singapore). These fresh nasal epithelial tissues were used to derive human nasal epithelial stem/progenitor cells (hNESPCs) and then differentiated into human nasal epithelial cells (hNECs) in air-liquid interface (ALI) culture system as described previously $[13,16,17]$. Briefly, primary cells were expanded with B-ALI ${ }^{\mathrm{sm}}$ complete growth medium (Lonza, Walkersville, MD) for about 1 week, and then transferred onto 12-well $0.4 \mu \mathrm{m}$ Transwell inserts (Corning, Corning, NY, USA). 4 days after seeding, growth medium was discarded and $700 \mu \mathrm{l}$ of PneumaCult ${ }^{\mathrm{tm}}$-ALI Medium with inducer supplements (STEMCELL Technologies Inc., Vancouver, British Columbia, Canada) was added to the basal chamber. The cells were cultured in ALI culture for 4 weeks, changing media every $2-3$ days, until they were fully differentiated.

\section{Viral infection of hNECs}

Fully differentiated hNECs were infected with human influenza A virus (IAV) H3N2 (Aichi/2/1968, American Type Culture Collection, Manassas, Va), strains of seasonal H1N1 (A/Singapore/G2-25.1/2014), H3N2 (A/ Singapore/G2-26.1/2014) and B/Victoria lineage (B/ Singapore/G2-14.1/2014) virus at a multiplicity of infection (MOI) of 0.1 and incubated at $35^{\circ} \mathrm{C}$ with $5 \% \mathrm{CO}_{2}$ for up to $72 \mathrm{~h}$ post infection (hpi), as described previously [13]. The cell lysate, supernatant and monocellular suspension were obtained at $8,16,24,48,72$ hpi. Uninfected controls were cultured in the same media without virus and were harvested at either 48 or $72 \mathrm{hpi}$.

\section{Poly $(\mathrm{I}: \mathrm{C})$ and UV-inactivated virus treatment}

Differentiated hNECs were also treated with $25 \mu \mathrm{g} / \mathrm{mL}$ poly (I:C) or same volume of media for 8,24 and $48 \mathrm{~h}$, and Ultraviolet light (UV) inactivated $\mathrm{H} 3 \mathrm{~N} 2$ virus or same media without virus for 24 and $48 \mathrm{~h}$ at the same MOI of 0.1.

\section{Plaque assay}

The cell supernatant was collected at set time-points by adding $150 \mu \mathrm{l} 1 \times \mathrm{dPBS}$ (Lonza, Walkersville, $\mathrm{Md}$ ) into the apical chamber and then incubated at $35^{\circ} \mathrm{C}$ for $10 \mathrm{~min}$. The cell supernatant was preserved at $-80^{\circ} \mathrm{C}$ until viral titration through plaque assay as described previously [13]. MDCK cells were cultured in 24-well plates overnight to $95 \%$ confluency. $100 \mu \mathrm{L}$ of serially diluted $\left(10^{-1}\right.$ to $\left.10^{-4}\right)$, TPCK-trypsin $(1 \mu \mathrm{g} / \mathrm{mL})$ activated virus samples were added and incubated for $1 \mathrm{~h}$ at $35^{\circ} \mathrm{C}$ where plates were rocked at $15 \mathrm{~min}$ interval. After incubation, the inoculums were discarded and $1 \mathrm{~mL}$ of $1.2 \%$ Avicel overlay (FMC BioPolymer, Philadelphia, Pa) was overlaid onto each well, then incubated for 65 to $72 \mathrm{~h}$ at $35^{\circ} \mathrm{C}$ with $5 \%$ $\mathrm{CO}_{2}$. At the end of incubation, Avicel overlay was then aspirated and cells were fixed in formaldehyde (4\% in $1 \times$ PBS) for $1 \mathrm{~h}$. Cells were then washed with $1 \times$ PBS, and $1 \%$ crystal violet was added for $15 \mathrm{~min}$ to stain cells. Plates 
were then rinsed with running water and air-dried. The PFU values were calculated from the plates as follows:

Number of plaques $\times$ Dilution factor $=$ Number of PFU per $100 \mu \mathrm{L}$.

\section{MUC15 siRNA transfection and infection}

Differentiated hNECs were transfected with $2 \mu \mathrm{M}$ nontargeting siRNA (NT-siRNA) or MUC15 siRNA in Accell siRNA delivery medium (GE Healthcare Dharmacon, Inc.) for 3 times at $-48,-24$ and 6 hpi. hNECs were then harvested at 24 and 48 hpi for RT-qPCR analysis.

\section{MUC15 recombinant protein treatment}

MDCK cells in 24-well (about $3 \times 10^{5}$ cells/well) were treated with human MUC15 recombinant protein (rMUC15, $24.14 \mathrm{kDa}$, LifeSpan BioSciences, Inc. Seattle, WA, USA) before or after the viral infection. For the pretreatment, $\mathrm{H} 3 \mathrm{~N} 2$ virus $\left(5 \times 10^{4} \mathrm{PFU} / 30 \mu \mathrm{l}\right)$ was incubated with 0,2 or $30 \mathrm{ng} \mathrm{rMUC15}$ in $30 \mu \mathrm{l}$ for $1 \mathrm{~h}$ on ice, followed by plaque assay on MDCK cells using the pretreated virus. For the post treatment, MDCK cells were infected with $\mathrm{H} 3 \mathrm{~N} 2$ virus at an MOI of 0.1 for $1 \mathrm{~h}$, where the inoculum was then removed and $500 \mu \mathrm{l}$ EMEM medium containing 0,2 or $30 \mathrm{ng}$ of rMUC15 and $1 \mu \mathrm{g} / \mathrm{mL}$ trypsin-TPCK was added. After incubation at $35^{\circ} \mathrm{C}$ for $48 \mathrm{~h}$, the culture supernatant was collected for plaque assay.

\section{RNA extraction, CDNA synthesis and qRT-PCR}

Total RNA isolation and cDNA synthesis were performed using mirVana miRNA isolation kit (Life Technologies) and Maxima First-Strand cDNA Synthesis Kit (Thermo Fisher Scientific) following the respective manufacturers' protocols. SYBR green-based qPCR analyses were conducted using specific primers purchased from SigmaAldrich (St Louis, Mo) (see Additional file 1: Table S1). All samples were tested in triplicate by using the GoTaqqPCR Master Mix kit (Promega, Madison, Wis), with thermal cycling parameters of $95^{\circ} \mathrm{C}, 15 \mathrm{~s} ; 60^{\circ} \mathrm{C}, 60 \mathrm{~s}$ each cycle for 40 cycles, at a thermal changing rate of $1.6^{\circ} \mathrm{C} / \mathrm{s}$ using ViiA 7 PCR machine (Applied Biosystems, Carlsbad, $\mathrm{CA})$. Mean cycle thresholds $(\mathrm{Ct})$ values were analyzed, and the relative gene expression normalized to housekeeping gene $P G K 1$ was calculated using the $2^{-\Delta \Delta \mathrm{Ct}}$ method.

\section{Western blotting}

Protein lysate of hNECs was harvested at various timepoints using RIPA lysis buffer (Thermo fisher) containing $1 \times$ protease and phosphatase inhibitor cocktail (Thermo fisher) following the manufacturer's instruction. After incubation on ice for $20 \mathrm{~min}$, the cell lysate was centrifuged at $13,200 \mathrm{rpm}$ for $20 \mathrm{~min}$ at $4{ }^{\circ} \mathrm{C}$ and then the supernatant was collected. Protein concentration was determined using BioRad protein assay (Bio-Rad, Hercules, Calif) according to the manufacturer's protocol and the absorbance at 595 $\mathrm{nm}$ was measured using a microplate reader (Synergy/HT, Biotek, Winooski, VT). $30 \mu \mathrm{g}$ of each protein sample was loaded and run with 10\% SDS-PAGE gel, and then transferred to PVDF membranes (Bio-Rad). Membranes was blocked with $5 \% \mathrm{w} / \mathrm{v}$ skim milk in $1 \times$ TBST (TRIS-buffered saline and $0.1 \% \mathrm{v} / \mathrm{v}$ Tween-20) at room temperature for $1 \mathrm{~h}$ and then incubated in the primary antibody solution against MUC15 (1:500 dilution, Abcam, Cambridge, UK), epithelial growth factor receptor (EGFR) (1:2000 dilution, Abcam), phospho-ERK (1:1000 dilution, Cell Signaling Technology), ERK (1:1000 dilution, Cell Signaling Technology), hemagglutinin (HA) (1:1000 dilution, Abcam), matrix protein 1 (M1), neuraminidase (NA) (1:1000 dilution, Sino Biological) and GAPDH (1:10000 dilution, Cell Signaling Technology) overnight at $4{ }^{\circ} \mathrm{C}$ and incubated in the HRPconjugated secondary anti-mouse IgG (1:5000 dilution, Cell Signaling Technology) or anti-rabbit IgG light chain (1: 2000, Abcam) for $1 \mathrm{~h}$ at room temperature. The ECL substrate was added (Cell Signaling Technology) to the blots and the blots were developed with scientific imaging films in the dark room. GAPDH was used to normalize sample loading. Semi-quantitative analysis was performed on the western blot bands where intensities of the bands were measured using image J.

\section{Immunoprecipitation}

$30 \mu \mathrm{l}$ of protein G-Sepharose beads (GE Healthcare, Singapore) were prepared by incubating in chilled lysis buffer containing $1 \mu \mathrm{g}$ of rabbit anti-MUC15 (ab171304, Abcam) or rabbit anti-IgG isotype control antibodies (ab27478, Abcam) and rotating for $2 \mathrm{~h}$ at $4{ }^{\circ} \mathrm{C}$. The beads were then washed thrice with ice-cold lysis buffer, followed by the addition of $10 \% \mathrm{BSA}$ and $300 \mu \mathrm{g}$ of cell lysates from H3N2-infected hNECs for $2 \mathrm{~h}$ at $4{ }^{\circ} \mathrm{C}$. Antibody-bound beads were heated at $95^{\circ} \mathrm{C}$ for $3 \mathrm{~min}$ and then centrifuged to obtain the immunoprecipitated proteins in the supernatant. The immunoprecipitated proteins were then analyzed using Western-blotting (10\% SDS-PAGE gel) with $10 \mu \mathrm{g}$ of total protein lysates used as input controls as described above.

\section{Cytospin and immunofluorescence staining}

At the stipulated time-points, hNECs were dissociated from Transwells with $1 \times$ Trypsin/EDTA solution (Gibco, Carlsbad, Calif). After fixation in $4 \%$ formaldehyde at room temperature for $10 \mathrm{~min}$, and dual $1 \times \mathrm{dPBS}$ wash, single-cell suspension via cytospin was prepared at a density of $1 \times 10^{4}$ cells/100 $\mu$ l using Shandon Cytospin 3 Cytocentrifuge (Thermo Fisher Scientific) at $500 \mathrm{rpm}$ for 5 min with mild acceleration.

The infected hNECs on the cytospin slides were permeabilized using $0.1 \%$ Triton X-100 at room temperature for $10 \mathrm{~min}$, and washed three times with milli Q water. Cells were then blocked using 10\% goat serum (DAKO, 
Glostrup, Denmark) for $30 \mathrm{~min}$ at room temperature, and incubated with primary antibody against MUC15 (1:50 dilution, Abcam) and HA (1:250, Abcam) in 1\% goat serum (DAKO) at $4{ }^{\circ} \mathrm{C}$ overnight. After incubation, cells were washed three times with $1 \times \mathrm{TBS}$, and incubated with fluorescent secondary antibody for $1 \mathrm{~h}$ at room temperature, followed by another three times of washing. Cells were then mounted using ProLong AntiFade reagent with DAPI and covered with microslides. Cytospin slides were observed using an Olympus IX51 fluorescence microscope under $40 \times$ objective lens or $100 \times$ oil lens.

\section{Statistical analysis}

Data were analyzed using GraphPad Prism 7 (GraphPad Software, La Jolla, Calif) software. For qPCR, the expression change of $M U C$ and host immune response genes was presented as fold change by normalizing with uninfected control in each group. Viral titer, mRNA or protein level was expressed as median and interquartile. Statistical significance was analyzed with one-way ANOVA and nonparametric, paired, Friedman test. $P$ values of less than .05 were considered significant. Cross-correlation between MUC15 mRNA level and the host immune response $\left(2^{-\Delta \Delta C t}\right)$, or protein level of MUC15 and EGFR (intensities of blot bands) was analyzed by using Spearman correlation test.

\section{Results}

MUC15 was the most consistently upregulated mucin during H3N2 infection among other mucins tested

To identify other $M U C$ genes involved during IAV H3N2 infection in hNECs, Transcriptomes from RNA sequencing and mRNA microarray of infected hNECs were analyzed (from different donors for each assays). Among 19 tested $M U C$ genes $(M U C 1,2,3 A, 3 B, 4,5 A C, 5 B, 6,7,8$, $12,13,15,16,17,19,20,21,22)$, significant changes of mRNA levels were observed for $M U C 1,3 A, 5 B, 13,15$ by RNA sequencing; while $M U C 3 A, 5 B, 15$ were found to be significantly altered in mRNA array at 48 hpi compared with uninfected controls, with consistent directional changes in expression. However, MUC5AC, the most common airway $M U C$ genes, did not show significant change of transcriptional expression (Fig. 1a), which is in line with our previous finding in influenza infection [13].

In order to confirm the transcriptional changes of these $M U C$ genes after H3N2 infection, RT-PCR was carried out using cDNA from influenza virus infected cells and uninfected controls of 13 patients. The results showed that mRNA expressions of MUC3A, 13 and 15 were significantly upregulated at 24 and 48 hpi compared with the uninfected controls (Fig. 1b). Interestingly, the expression change of MUC15 gene expression was the most significant and this increasing change was consistent across almost every hNEC sample from different donors (see Additional file 2: Table S2). In addition, we tested MUC15 gene expression in other seasonal influenza virus strains (H1N1, H3N2 and B) and observed consistent upregulation across influenza strains (Additional file 3: Figure S1), a phenomenon not observed in $M U C 13$ and $M U C 3 A$. Therefore, we selected $M U C 15$ for further downstream investigations.

In addition, the protein expression of MUC15 was also upregulated upon $\mathrm{H} 3 \mathrm{~N} 2$ infection as demonstrated using western blotting (Fig. 1c and d). The immunofluorescence co-staining of MUC15 and influenza viral hemagglutinin (HA) showed that MUC15 was tethered on the cell plasma membrane, and that viral infection significantly increased MUC15 expression (Fig. 1e and Additional file 4: Figure S2). Co-staining of MUC15 and viral HA prompted us to further explore if there was any interaction between MUC15 and influenza surface protein in view of the membrane expression of MUC15.

\section{MUC15 up-regulation was induced only with active infection; and did not interfere with viral replication}

IAV H3N2, UV-inactivated H3N2 and $25 \mu \mathrm{g} / \mathrm{mL}$ of poly (I:C) were used to treat fully differentiated hNECs to assess its upregulation in response to active, inactive and viral components. UV exposure successfully eliminated ability of viral replication as viral titers could be only detected in the H3N2 infected group but not in uninfected control or the UV-inactivated $\mathrm{H} 3 \mathrm{~N} 2$ treated group (Fig. 2a). Similarly, both transcriptional and translational expression of MUC15 increased only in H3N2 infected hNECs, but not in UV-inactivated group and poly (I:C) group (Fig. 2b and c).

Although significant change of $M U C 15$ expression upon viral replication was elucidated, $M U C 15$ gene knockdown did not interfere with viral replication (Fig. 2d). Further experiments using human recombinant MUC15 protein (rMUC15) showed that MUC15 did not interact with or neutralize H3N2 virus. This is shown by the absence of significant changes in viral titer between rMUC15-treated group and the control, regardless of treatment before or after infection (Fig. 2e). The same experiment was carried out on hNECs in ALI culture and the result indicated that rMUC15 could not inhibit viral entry into hNECs or diminish viral replication (Fig. 2f). Furthermore, co-IP assay indicated no interaction between MUC15 and H3N2 viral proteins (HA, NA and M1; Fig. 2g).

\section{Expression of MUC15 was positively correlated with inflammatory factors}

Since $M U C 15$ failed to hinder viral entry and viral replication of H3N2, we correlated its expression with the host immune responses induced after H3N2 infection of hNECs. Inflammatory cytokines and chemokines including $I L 1 B$, IL6, IL8, TNF, IFNB1, CCL2 and CXCL10 were increased 


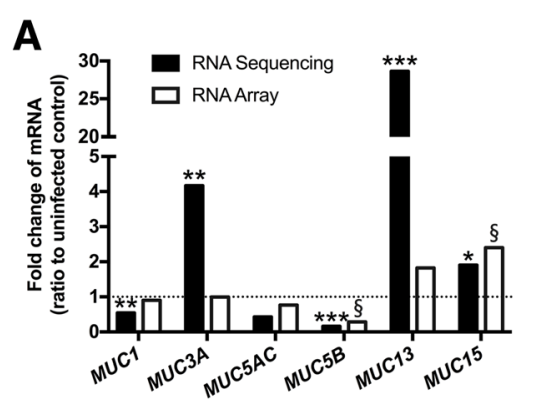

\section{C}

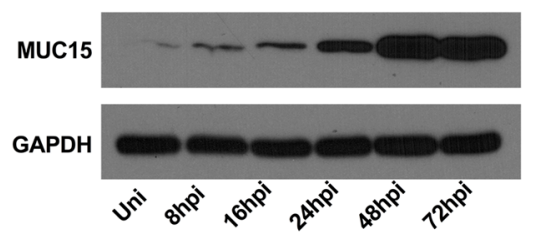

B
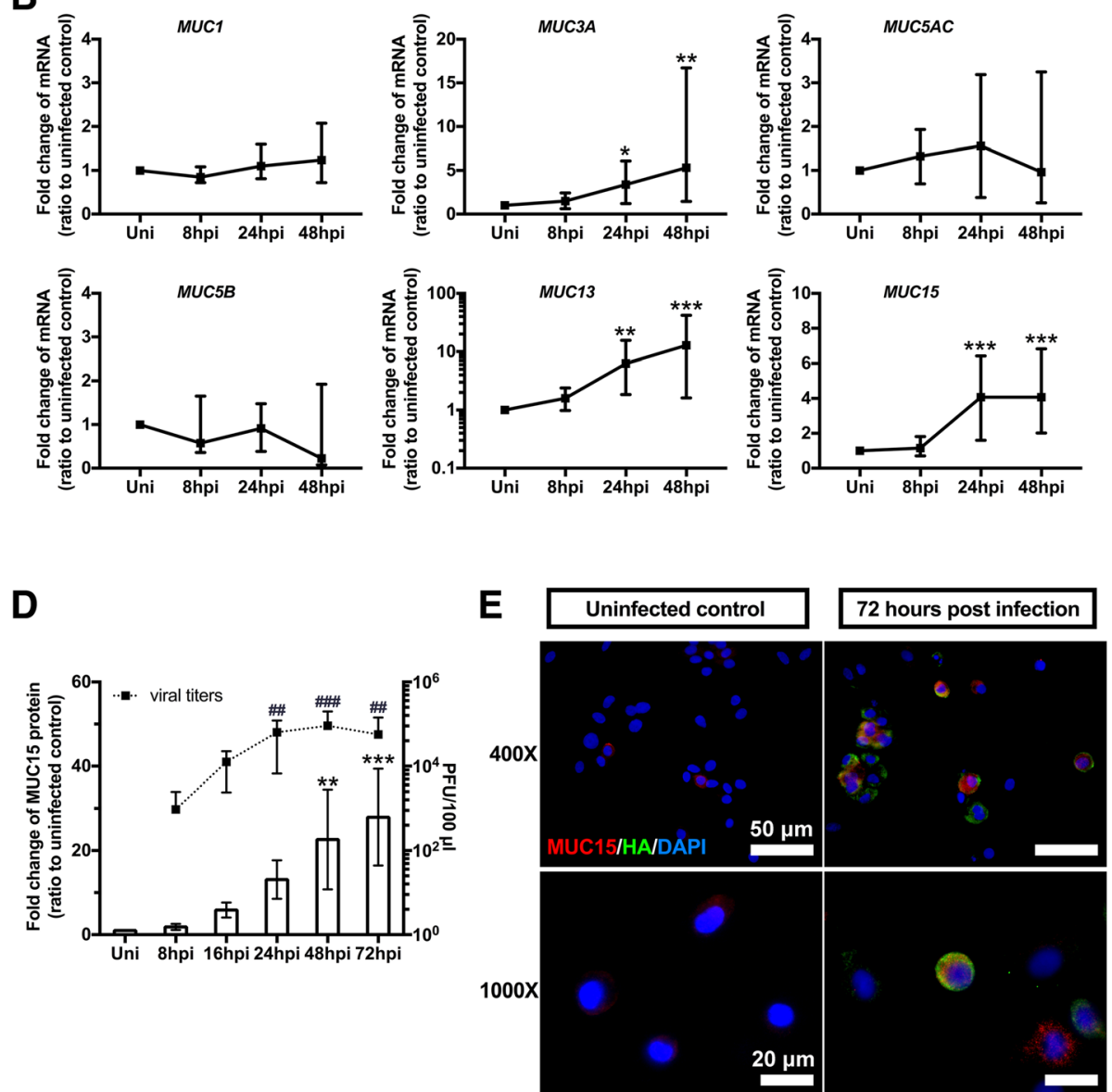

Fig. 1 MUC15 was upregulated after H3N2 infection in hNECs a: Fold changes of MUC mRNA expression in hNECs infected by H3N2 at $48 \mathrm{~h}$ post infection (hpi) with uninfected subjects (Uni) as baseline, detected by RNA sequencing (black column, $n=3$ ) and mRNA array (white column, $n=$ 5). b: Quantitative real-time RT-PCR analysis of MUC genes in hNECs infected with H3N2 with uninfected control subjects as baseline ( $n=13)$,

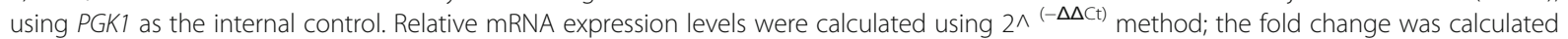
compared against uninfected control subjects. c: Western blot analysis of MUC15 protein expression on hNECs infected with H3N2. Cells were harvested at various times as indicated. $\mathbf{d}$ : The western blot band intensities were measured using Image $\mathrm{J}$, and graph is presented as the ratio of MUC15-to-GAPDH and then normalized to each uninfected control $(n=5)$ (left y axis and bars). Plaque assay show viral replication in hNECs infected by H3N2 ( $n=8$ ) (right y axis and spots). e: IF co-staining of MUC15 (red) and HA (green) on fully differentiated hNECs with or without

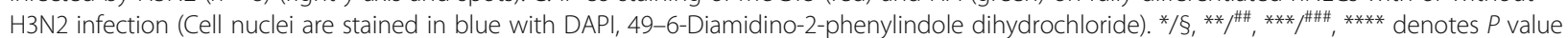
of less than $0.05,0.01,0.001,<0.0001$ compared with uninfected control, respectively. Median values with 25 th and 75 th percentiles are indicated by error bar. Uni: uninfected control

both at mRNA levels (Fig. 3a) and protein levels as previously demonstrated [13]. Besides, there were positive correlations between mRNA levels of MUC15 versus these inflammatory factors (Fig. 3b). Interestingly, the mRNA levels of most factors showed peak or plateauing trend at time-points coinciding with the increasing trend of MUC15. Additional MUC15 knockdown experiments in hNECs showed increase in immune responses (IFN $\beta$ and 
A

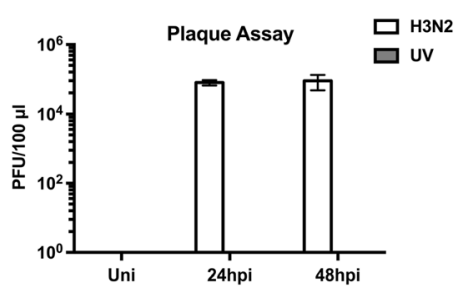

C

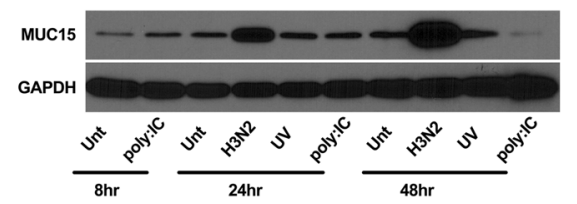

B

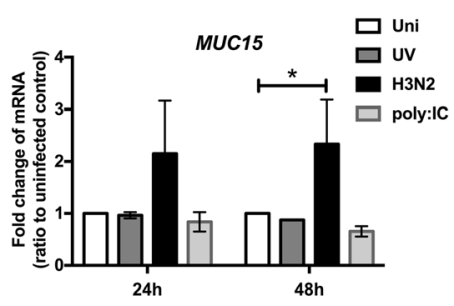

D

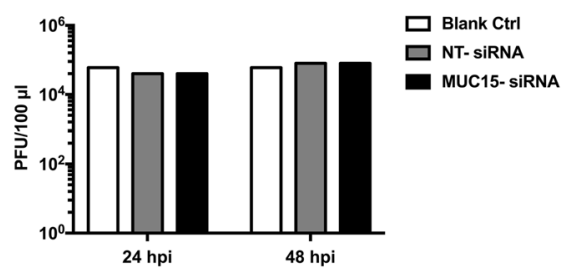

E

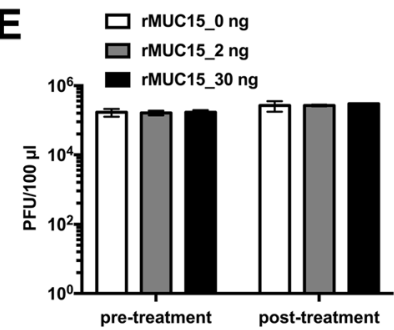

$\mathbf{F}$

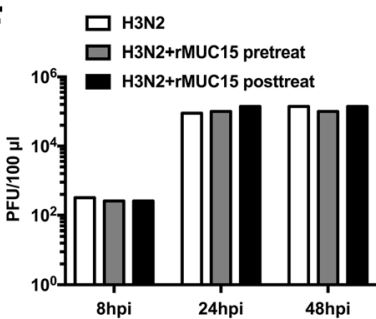

G

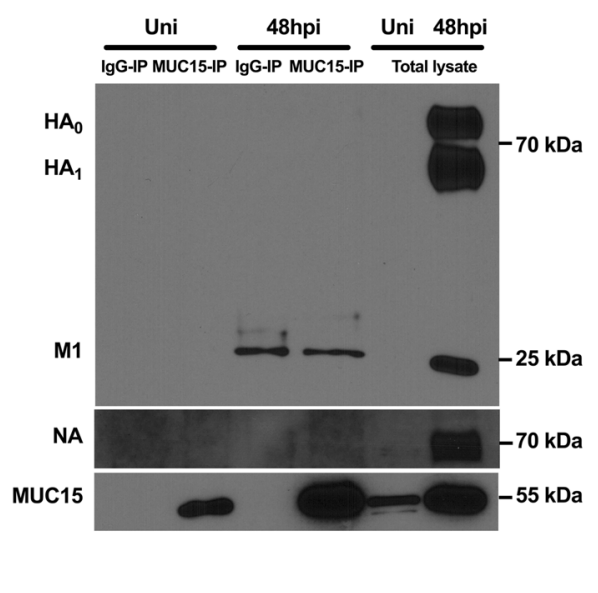

Fig. 2 Active viral replication induced MUC15 upregulation but MUC15 did not interfere with viral replication a: Plaque assay showed viral replication in hNECs with or without H3N2 infection or UV-inactivated H3N2 (UV) $(n=2)$. b: Quantitative real-time RT-PCR analysis of MUC15 mRNA expression in hNECs treated with or without H3N2, UV-inactivated H3N2 (UV) or $25 \mu \mathrm{g} / \mathrm{mL}$ poly (I:C) for 24 and $48 \mathrm{~h}$ with uninfected control subjects as baseline $(n=2)$, using PGK1 as the internal control. Relative mRNA expression levels were calculated using $2 \wedge(-\Delta \Delta \mathrm{Ct})$ method; the fold change was compared against uninfected control subjects. c: Western blot analysis of MUC15 protein expression on hNECs treated with or without H3N2, UV-inactivated H3N2 (UV) or $25 \mu \mathrm{g} / \mathrm{mL}$ poly (l:C). Cells were harvested at various times as indicated $(n=2)$ ). d: Viral replication in hNECs transfected with non-targeting siRNA (NT-siRNA) or MUC15-siRNA and normal hNECs $(n=1)$. e-f: Viral replication in MDCK cells (E, $n=3$ ) and hNECs $(F, n=1)$ treated with 0,2 or 30 ng recombinant MUC15 protein (rMUC15) before or after H3N2 infection. g: Immunoprecipitation with MUC15 protein followed by Western blotting for hemagglutinin (HA), matrix protein 1 (M1), neuraminidase (NA) and MUC15 ( $n=2)$. Median values with 25 th and 75 th percentiles are indicated by error bar. ${ }^{*}$ denotes $P$ value of less than 0.05 compared with uninfected control. Uni: uninfected control; Unt: untreated control

CXCL10) even at weak MUC15 reduction (Additional file 5: Figure S3). In addition, we detected reducing trends in expression of epithelial growth factor receptor (EGFR), ERK1/ 2 and pERK1/2, which are previously reported targets of MUC15 $[18,19]$. In response to H3N2 infection, we observed that the protein expression of EGFR was downregulated during $\mathrm{H} 3 \mathrm{~N} 2$ infection in hNECs, which was negatively correlated with that of MUC15 (Fig. 4a and Fig. 4b). Besides, ERK1/2 and phosphorylated ERK1/2 were found to increase after H3N2 infection in hNECs and then decreased at $72 \mathrm{hpi}$, which coincided with increased expression of MUC15 (Fig. 4c). Downstream of EGFR-pERK/ERK signaling, the transcription factor AP1 (JUN and FOS) was downregulated as well (Fig. 4d and e). Taken together, these findings suggested an interaction between MUC15 and immunomodulatory factors late into infection with H3N2 virus, and warrants further investigation to establish their exact immunomodulatory mechanisms.

\section{Discussion}

The cell-surface mucins have been explored for their role in transduction of cellular signals, modulation of cell proliferation-differentiation and regulation of host immune response to microbial infections $[3,20]$. Other than 


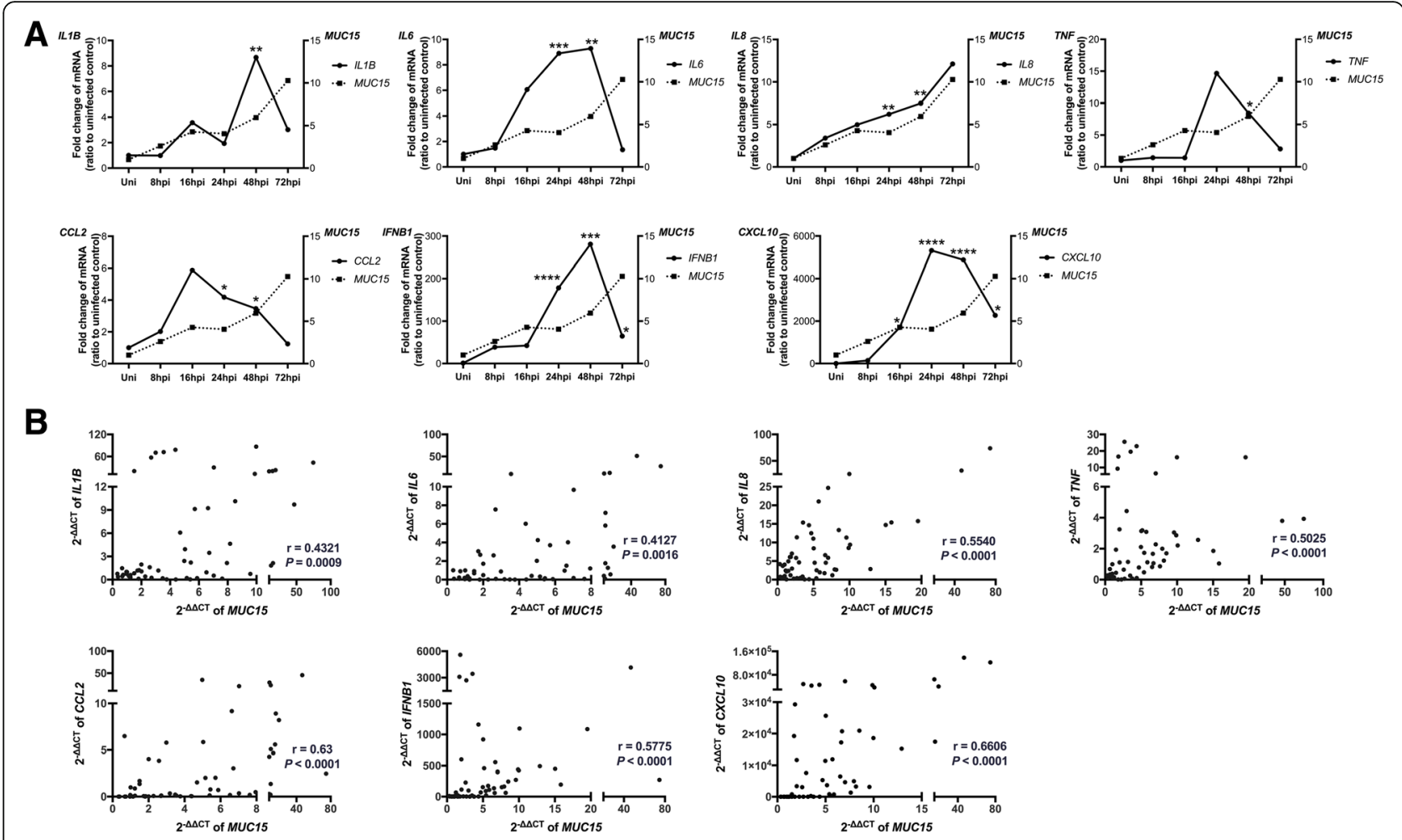

Fig. 3 Expression of MUC15 was positively correlated with inflammatory factors a: Quantitative real-time RT-PCR analysis of IL1B, IL6, IL8, TNF, CCL2, IFNB1, CXCL10, (solid line, left y axis) and MUC15 (dotted line, right y axis) mRNA expression in hNECs infected with H3N2 ( $n=11$ ), using PGK1 as the internal control. Relative mRNA expression levels were calculated using $2 \wedge^{(-\Delta \Delta \mathrm{Ct})}$ method; the fold change was compared against uninfected control subjects. b: The correlations between mRNA level $\left(2 \wedge^{(-\Delta \Delta C t)}\right)$ of MUC15 versus IL1B, IL6, IL8, TNF, CCL2, IFNB1, CXCL10 in hNECs infected with H3N2 were analyzed $(n=11)$. The timepoints post-infection that were included in the graphs are $8,16,24,48$ and 72 hpi. ****,**, **** denotes $P$ value of less than $0.05,0.01,0.001,<0.0001$ compared with uninfected control, respectively. Uni: uninfected control

MUC1, levels of MUC4 and MUC16 were also significantly increased in inflamed conditions such as in COPD patients [21, 22]. With respect to this evidence, cellsurface mucins may play an important role in modulation of infection and inflammation. Acute excessive inflammation will impact the host when unregulated, from epithelium injury to systemic inflammatory response syndrome (SIRS), organ failure and even death. Besides, prolonged pathological inflammation can also result in chronic inflammatory diseases such as asthma and chronic obstructive pulmonary disease (COPD). Among these cell surface mucins, MUC1 is implicated in the pathogenesis of influenza infection [12]. Therefore, it is of great clinical significance to further identify if there are other cell-surface mucins involved in the pathogenesis of influenza infection, i.e. for their utility as markers or targets for influenza management.

In this study, we found that MUC15 mRNA and protein expression was upregulated after IAV H1N1, H3N2 and influenza B infection in human nasal epithelial cells. MUC15 is a newly reported glycosylated protein with structural hallmarks of other membrane-tethered mucins, with an extracellular region with several potential glycosylation sites, a putative transmembrane domain, and a short cytoplasmic C-terminal [23]. The few studies on MUC15 focused on its association with genesis of carcinoma [18, 19, 24-26]. Although mRNA level of MUC15 can be induced by respiratory syncytial virus (RSV) and human metapneumovirus (hMPV) infection in human epithelial cells [27], MUC15 expression and function upon influenza virus infection are yet unclear. In addition, MUC1 is the first cell-surface mucin with anti-influenza function [12], and it was reported that MUC1 and MUC15 in the milk-fat globule membrane milk can limit bacterial and viral infections of the gastrointestinal tract [28-30]. Therefore, in view of its consistent elevation across different hNEC samples and influenza strains, we assessed the effects of MUC15 changes following influenza infection. Unlike MUC1 however, increase of this cell-surface mucin did not interfere with viral entry into epithelial cells by directly interacting with virus particles. Correlations of MUC15 mRNA levels versus many immunological factors responding to influenza infection, have been revealed, and may be important for future studies to elucidate MUC15 immunomodulatory functions. Besides, we also 


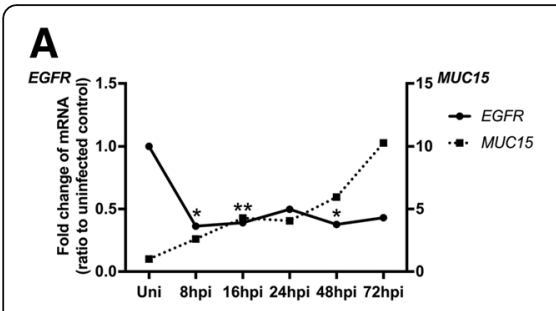

D
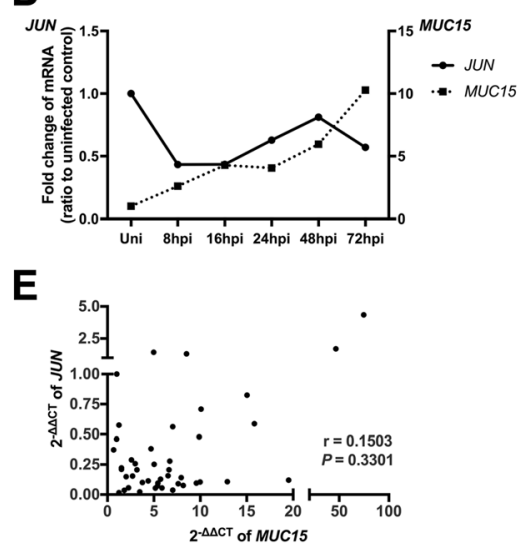
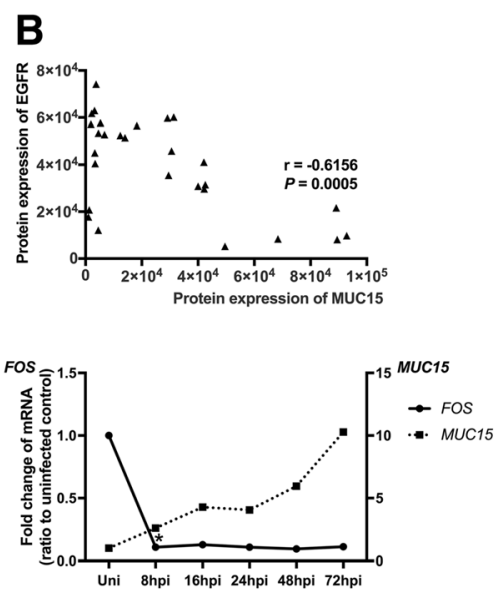

Uni 8 hi 16 hpi 24 pi 48 pi 72 hpi

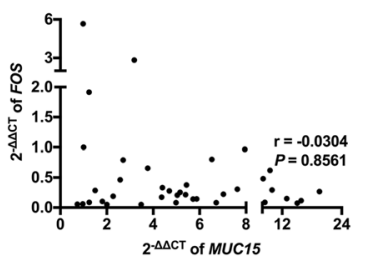

C

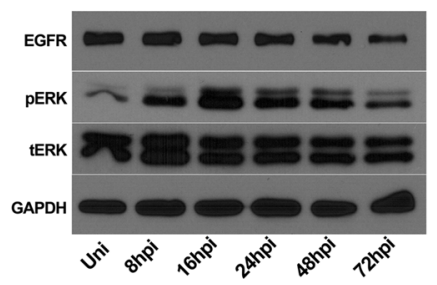

$\mathbf{F}$

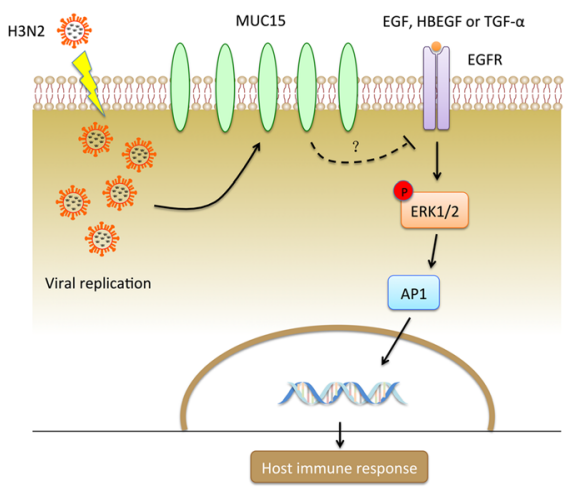

Fig. 4 EGFR-pERK1/2-AP1 pathway was triggered by viral infection. a: Quantitative real-time RT-PCR analysis of EGFR (solid line, left y axis) and MUC15 (dotted line, right y axis) mRNA expression in hNECs infected with H3N2 $(n=11)$, using PGK1 as the internal control. Relative mRNA expression levels were calculated using $2{ }^{\wedge}{ }^{(-\Delta \Delta C t)}$ method; the fold change was compared against uninfected control subjects. $\mathbf{b}$ : The correlations between protein level (blot band intensities) of MUC15 versus EGFR in hNECs with or without H3N2 infection were analyzed $(n=5)$. c: Western blot analysis of EGFR, pERK, total ERK and GAPDH protein expression on hNECs with or without H3N2infection. Cells were harvested at various times as indicated $(n=5)$. d: Quantitative real-time RT-PCR analysis of JUN and FOS mRNA expressions in hNECs with or without H3N2 infection $(n=11)$, using $P G K 1$ as the internal control. Relative mRNA expression levels were calculated using $2^{\wedge}{ }^{(-\Delta \Delta C t)}$ method; the fold change was compared against uninfected control subjects. e: The correlations between mRNA level $\left(2^{\wedge}{ }^{(-\Delta \triangle \mathrm{Ct})}\right)$ of MUC15 versus JUN and FOS in hNECS infected with H3N2 were analyzed $(n=11)$. f: The hypothesis of MUC15's potential role in hNECs infected with H3N2: The increase of MUC15 expression triggered by H3N2 viral replication inhibits the activation of epithelial growth factor receptor (EGFR), which initiates the downstream ERK1/2-AP1 pathway. Thus, MUC15 may downregulate the host immune response at the later phase of viral infection. ${ }^{*},{ }^{* *}, *^{* * *},{ }^{* * *}$ denotes $P$ value of less than $0.05,0.01,0.001,<0.0001$ compared with uninfected control, respectively. Uni: uninfected control

detected two main cellular signaling factors - EGFR and phosphorylated ERK1/2 (found as MUC15 targets), which could be stimulated in response to viral infection, and may induce downstream reactions such as anti-viral cytokine production. We also observed that expression of EGFR and phosphorylation of ERK1/2 were reduced at late phase of viral infection (such as $48 \mathrm{hpi}, 72 \mathrm{hpi}$ ), in response to MUC15 increase. In this respect, MUC15 may play an immunomodulatory role in human nasal epithelial cells infected by H3N2 through regulation of the EGFR-pERK1/2-AP1 pathway (Fig. 4f).

Wang et al. revealed that MUC15 could bind EGFR at the cell membrane to block the dimerization and activation of EGFR [19]. Thus, MUC15 could potentially exert its immunomodulatory functions via inhibition EGFR and its downstream pathway. On the other hand, MAPK pathway is a crucial signaling pathway upon viral infection. Ligands such as EGF, HBEGF, and TGF- $\alpha$ would stimulate EGFR to activate ERK1/2. Activator protein (AP1) is a heterodimer composed of c-Fos and c-Jun, whose transcriptional activity would be enhanced by phosphorylation of ERK1/2, and then promote the expression of IFN- $\beta$ and anti-viral cytokines [31-33]. If MAPK pathway activation is reduced, anti-viral inflammation could be also suppressed, preventing over-inflammation to mitigate inflammatory damage to the host. In our study with hNECs, no direct interaction between MUC15 and EGFR was observed (data not shown); while treatment of rMUC15 protein also did not significantly reduce EGFR expression (data not shown). However, we observed decreased levels of EGFR, ERK and pERK in the late phase of infection, which coincided with strong induction of MUC15 in the nasal epithelium, indicating its potential role in immunemodulation, possibly via other indirect means.

Airway epithelium is a component of the innate immune system and its abnormal response to the pathogens or allergens may result in the genesis of chronic airway diseases. In recent years, some researchers 
have found that there was a strong link between early acute viral infection and subsequent chronic airway diseases [34, 35]. Furthermore, acute viral infection is also a common risk factor for the exacerbation of chronic airway diseases [35, 36]. Virus-derived inflammation clearly plays a central role in these processes. For instance, interleukin-1 $\beta$ (IL-1 $\beta$ ) and interleukin17A (IL-17A) may mediate neutrophilic inflammation in influenza-initiated exacerbation of chronic lung inflammation [37]. As a counterpart of MUC1, MUC15 was upregulated by several respiratory viruses, including influenza, as showed in our results, respiratory syncytial virus (RSV) and human metapneumovirus (hMPV). This increased expression occurred at the late phase of infection (48 or $72 \mathrm{hpi}$ ) [27], while most of virus-induced cytokines and chemokines peaked and plateaued. Therefore, we suspect that the increased MUC15 level may be in response to the activation of these factors and may play a role in their regulation.

Another interesting finding was that the upregulation of MUC15 expression only occurred during active infection of the nasal epithelial cells, rather than by UVinactivated virus or viral mimics like poly (I:C). This indicated that increased MUC15 might be induced by the virus as a defense mechanism against host inflammatory responses. Future work can therefore focus on the interaction between the influenza NS1 protein and MUC15, and as a possible diagnosticbiomarker for severe influenza infections.

\section{Limitations}

The main limitation in this study is that we could not ascertain the anti-inflammatory role of MUC15 during viral infection through the MUC15 knockdown experiments, because the hNECs derived from nasal biopsies were poorly transfected. Attempts at siRNA transfection on hNECs yielded MUC15 knockdown in the absence of infection, but was weaker during infection due to the high levels of MUC15 expression and activation, albeit the increase of IFN $\beta$ and CXCL10 was observed. On the other hand, airway epithelial cell lines such as A549 cells expressed lower levels of MUC15 compared to hNECs and were thus not used for knockdown experiments (data not shown). Hence, in view of the interesting finding of MUC15 induction in hNECs, future studies on mechanistic investigation of MUC15 could include gene editing to completely knockout MUC15 to observe its loss of function effect following influenza infection, particularly the effects on innate immune responses. Gene editing studies on cell systems such as hNECs have been recently successfully explored, and can potentially be implemented for studies of cell surface mucins [38].

\section{Conclusion}

The cell-surface mucin MUC15 was upregulated in hNECs after IAV H3N2 infection. Despite the lack of direct interaction with the virus to inhibit viral entry or viral replication, MUC15 showed a positive correlation with inflammatory factors at the level of mRNA expression. Therefore, it is possible that MUC15 may play an immunomodulatory role during $\mathrm{H} 3 \mathrm{~N} 2$ infection in the regulation of inflammatory responses. Nevertheless, in view of the strong induction of MUC15 following influenza infection of multiple strains, MUC15 may be exploited as a novel biomarker of influenza infection, and its functions during infection should be fully explored in the future.

\section{Additional files}

Additional file 1: Table S1. Sequence of Primers for Real-Time Polymerase Chain Reaction. (DOCX 15 kb)

Additional file 2: Table S2. Fold change of MUC15 mRNA expression. (DOCX $14 \mathrm{~kb}$ )

Additional file 3: Figure S1. Expression of MUC15, MUC13 and MUC3A in seasonal influenza H1N1, H3N2 and B infection. Quantitative real-time RT-PCR analysis of MUC15, MUC13 and MUC3A gene mRNA expression in hNECs infected with seasonal influenza H1N1, H3N2, and B, with uninfected control subjects as baseline $(n=4)$ and using PGK1 as the internal control. Relative mRNA expression levels were calculated using $2 \wedge^{(-\Delta \Delta C t)}$ method; the fold change was calculated as fold increases from uninfected control subjects. * and ${ }^{* *}$ denotes $P$ value of less than 0.05 and 0.01 compared with uninfected control, respectively. (PDF 37 kb)

Additional file 4: Figure S2. Mean fluorescence intensity (MFI) of MUC15 in infected hNECs. Five different images were captured from every slide and the mean immunofluorescence intensities (MFI) of MUC15 were measured using Image J $(n=5)$. Data was then normalized to each uninfected control. ${ }^{*},{ }^{* *},{ }^{* *},{ }^{* * * *}$ denotes $P$ value of less than $0.05,0.01,0.001,<0.0001$ compared with uninfected control, respectively. Median values with 25 th and 75 th percentiles are indicated by error bar. Uni: uninfected control. (PDF $21 \mathrm{~kb}$ )

Additional file 5: Figure S3. Expression of MUC15, IFN $\beta$ and CXCL10 in MUC15 siRNA knockdown in hNECs. siRNA knockdown of MUC15 in hNECs $(n=1)$. siRNA knockdown of MUC15 was achieved stronger in non-infected hNECs (both 24 and 48 hpi). Conversely, the knockdown was greatly reduced in infected hNECs when MUC15 is highly induced; and the knockdown is only slightly achieved at 24 hpi (grey bars). Quantitative real-time RT-PCR analysis of MUC15, IFN $\beta$ and CXCL10 was performed using $P G K 1$ as internal control. Relative mRNA expression levels were calculated using $2 \wedge^{(-\Delta \Delta C t)}$ method. Hpi: hours post infection; NT: non-targeting control; M15: MUC15 siRNA. (PDF 41 kb)

\section{Abbreviations}

ALI: Air-liquid interface; AP1: Activator protein 1; COPD: Chronic obstructive pulmonary disease; EGFR: Epithelial growth factor receptor;

HA: Hemagglutinin; hMPV: human metapneumovirus; hNECs: human nasal epithelial cells; hNESPCs: human nasal epithelial stem/progenitor cells; hpi: hours post infection; IAV: Influenza A virus; IL-17A: Interleukin-17A; IL-

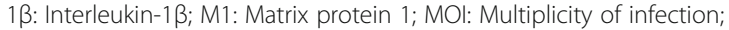
NA: Neuraminidase; PCL: Periciliary layer; PGK1: Phosphoglycerate kinase 1; rMUC15: human MUC15 recombinant protein; RSV: Respiratory syncytial virus; SIRS: Systemic inflammatory response syndrome; TLR: Toll-like receptor; UV: Ultraviolet light

\section{Acknowledgements}

We acknowledge Dr. Yongkang Qiao for her advice on the coImmunoprecipitation assay. 


\section{Authors' contributions}

ZGC and ZNW designed the experiments. ZNW performed the experiments and wrote the manuscript. $Y Y, J L$ and $T T H$ assisted with performing the experiments. KTT and YKO collected the human samples. VTKC and DYW contributed to experimental design and intellectual input. KST contributed significantly to the experimental design, conducted the virus infection and manuscript preparation. All authors have read and approved the manuscript.

\section{Funding}

This study was supported by grants from the National Medical Research Council [NMRC/CIRG/1458/2016] and the National Natural Science Foundation of China [81470219]. These grants were awarded to Prof. De Yun Wang and Prof. Zhuang Gui Chen, respectively. Prof. Zhuang Gui Chen contributed to designing this study and Prof. De Yun Wang contributed to experimental design and intellectual input.

\section{Availability of data and materials}

The datasets used and/or analyzed during the current study available from the corresponding author on reasonable request.

\section{Ethics approval and consent to participate}

This study was performed in accordance with the Declaration of Helsinki and had been approved by the National Healthcare Group Domain-Specific Review Board of Singapore (Ethics approval number: DSRB D/11/228; IRB 13509). Written consent has been obtained from study participants after detailed explanation of the study.

\section{Consent for publication}

Not applicable.

\section{Competing interests}

The authors declare that they have no competing interests.

\section{Author details}

${ }^{1}$ Department of Pediatrics, The Third Affiliated Hospital of Sun Yat-sen University, Guangzhou, Guangdong, China. ${ }^{2}$ Department of Otolaryngology Yong Loo Lin School of Medicine, National University of Singapore, 1 E Kent Ridge Road, Singapore 119228, Singapore. ${ }^{3}$ Center for Interventional Medicine, The Fifth Affiliated Hospital of Sun Yat-sen University, Zhuhai, Guangdong, China. ${ }^{4}$ Department of Microbiology and Immunology, Yong Loo Lin School of Medicine, National University of Singapore, Singapore, Singapore.

\section{Received: 18 February 2019 Accepted: 23 June 2019} Published online: 15 July 2019

\section{References}

1. Vareille M, Kieninger E, Edwards MR, Regamey N. The airway epithelium: soldier in the fight against respiratory viruses. Clin Microbiol Rev. 2011;24(1): 210-29. https://doi.org/10.1128/CMR.00014-10.

2. Nicholas B, Skipp P, Mould R, et al. Shotgun proteomic analysis of human-induced sputum. Proteomics. 2006;6:4390-401. https://doi.org/10. 1002/pmic.200600011.

3. Lillehoj EP, Kato K, Lu W, Kim KC. Cellular and molecular biology of airway mucins. Int Rev Cell Mol Biol. 2013;303:139-202. https://doi.org/10.1016/ B978-0-12-407697-6.00004-0.

4. Fahy JV, Dickey BF. Airway mucus function and dysfunction. N Engl J Med. 2010;363(23):2233-47. https://doi.org/10.1056/NEJMra0910061.

5. Matsui H, Grubb BR, Tarran R, et al. Evidence for periciliary liquid laye depletion, not abnormal ion composition, in the pathogenesis of cystic fibrosis airways disease. Cell. 1998;95(7):1005-15.

6. Kesimer M, Ehre C, Burns KA, Davis CW, Sheehan JK, Pickles RJ. Molecular organization of the mucins and glycocalyx underlying mucus transport over mucosal surfaces of the airways. Mucosal Immunol. 2013;6(2):379-92. https://doi.org/10.1038/mi.2012.81.

7. Singh PK, Hollingsworth MA. Cell surface-associated mucins in signal transduction. Trends Cell Biol. 2006;16(9):467-76. https://doi.org/10.1016/ j.tcb.2006.07.006.

8. Lillehoj EP, Hyun SW, Kim BT, et al. Muc1 mucins on the cell surface are adhesion sites for Pseudomonas aeruginosa. Am J Physiol Lung Cell Mol Physiol. 2001;280:L181-7. https://doi.org/10.1152/ajplung.2001.280.1.L181.
9. Ueno K, Koga T, Kato K, et al. MUC1 mucin is a negative regulator of toll-like receptor signaling. Am J Respir Cell Mol Biol. 2008;38(3):263-8. https://doi. org/10.1165/rcmb.2007-0336RC.

10. Li Y, Dinwiddie DL, Harrod KS, Jiang Y, Kim KC. Antiinflammatory effect of MUC1 during respiratory syncytial virus infection of lung epithelial cells in vitro. Am J Physiol Lung Cell Mol Physiol. 2010;298(4):L558-63. https://doi. org/10.1152/ajplung.00225.2009.

11. Kyo Y, Kato K, Park YS, et al. Anti-inflammatory role of MUC1 mucin during infection with nontypeable Haemophilus influenzae. Am J Respir Cell Mol Biol. 2012;46(2):149-56. https://doi.org/10.1165/rcmb.201101420C.

12. McAuley $\mathrm{L}$, Corcilius L, Tan HX, Payne RJ, McGuckin MA, Brown LE. The cell surface mucin MUC1 limits the severity of influenza a virus infection. Mucosal Immunol. 2017;10(6):1581-93. https://doi.org/10.1038/mi.2017.16.

13. Yan Y, Tan KS, Li C, et al. Human nasal epithelial cells derived from multiple subjects exhibit differential responses to $\mathrm{H} 3 \mathrm{~N} 2$ influenza virus infection in vitro. J Allergy Clin Immunol. 2016;138(1):276-281.e15. https://doi.org/10. 1016/j.jaci.2015.11.016.

14. Qiao Y, Yan Y, Tan KS, et al. CD151, a novel host factor of nuclear export signaling in influenza virus infection. J Allergy Clin Immunol. 2018;141(5): 1799-817. https://doi.org/10.1016/j.jaci.2017.11.032.

15. Tian T, Zi X, Peng Y, et al. H3N2 influenza virus infection enhances oncostatin M expression in human nasal epithelium. Exp Cell Res. 2018; 371(2):322-9. https://doi.org/10.1016/j.yexcr.2018.08.022.

16. Li YY, Li CW, Chao SS, et al. Impairment of cilia architecture and ciliogenesis in hyperplastic nasal epithelium from nasal polyps. J Allergy Clin Immunol. 2014;134(6):1282-92. https://doi.org/10.1016/j.jaci.2014.07.038.

17. Zhao X, Yu F, Li C, et al. The use of nasal epithelial stem/progenitor cells to produce functioning ciliated cells in vitro. Am J Rhinol Allergy. 2012;26(5): 345-50. https://doi.org/10.2500/ajra.2012.26.3794.

18. Huang J, Che Ml, Huang YT, et al. Overexpression of MUC15 activates extracellular signal-regulated kinase $1 / 2$ and promotes the oncogenic potential of human colon cancer cells. Carcinogenesis. 2009;30(8):1452-8. https://doi.org/10.1093/carcin/bgp137.

19. Wang RY, Chen L, Chen HY, et al. MUC15 inhibits dimerization of EGFR and PI3K-AKT signaling and is associated with aggressive hepatocellular carcinomas in patients. Gastroenterology. 2013;145(6):1436-48. https://doi. org/10.1053/j.gastro.2013.08.009.

20. Apostolopoulos V, Stojanovska L, Gargosky SE. MUC1 (CD227): a multitasked molecule. Cell Mol Life Sci. 2015;72(23):4475-500. https://doi.org/10. 1007/s00018-015-2014-z

21. Fischer BM, Cuellar JG, Diehl ML, deFreytas AM, Zhang J, Carraway KL, Voynow JA. Neutrophil elastase increases MUC4 expression in normal human bronchial epithelial cells. Am J Physiol Lung Cell Mol Physiol. 2003; 284(4):L671-9. https://doi.org/10.1152/ajplung.00220.2002.

22. Yilmaz MB, Zorlu A, Dogan OT, Karahan O, Tandogan I, Akkurt I. Role of CA125 in identification of right ventricular failure in chronic obstructive pulmonary disease. Clin Cardiol. 2011;34(4):244-8. https://doi.org/10.1002/ clc.20868 Epub 2011 Mar 14.

23. Pallesen LT, Berglund L, Rasmussen LK, Petersen TE, Rasmussen JT. Isolation and characterization of MUC15, a novel cell membrane-associated mucin. Eur J Biochem. 2002;269(11):2755-63.

24. Nam KH, Noh TW, Chung SH, et al. Expression of the membrane mucins MUC4 and MUC15, potential markers of malignancy and prognosis, in papillary thyroid carcinoma. Thyroid. 2011;21(7):745-50. https://doi.org/10. 1089/thy.2010.0339.

25. Yang WB, Li CY. Correlations of MUC15 overexpression with clinicopathological features and prognosis of glioma. J Huazhong Univ Sci Technolog Med Sci. 2014;34(2):254-9. https://doi.org/10.1007/s11596014-1267-3.

26. Oh HR, An CH, Yoo NJ, Lee SH. Frameshift mutations of MUC15 gene in gastric and its regional heterogeneity in gastric and colorectal cancers. Pathol Oncol Res. 2015;21(3):713-8. https://doi.org/10.1007/ s12253-014-9878-3.

27. Baños-Lara MDR, Piao B, Guerrero-Plata A. Differential mucin expression by respiratory syncytial virus and human Metapneumovirus infection in human epithelial cells. Mediat Inflamm. 2015;2015:347292. https://doi. org/10.1155/2015/347292.

28. Peterson JA, Patton S, Hamosh M. Glycoproteins of the human milk fat globule in the protection of the breast-fed infant against infections. Biol Neonate. 1998;74:143-62. 
29. Yolken RH, Peterson JA, Vonderfecht SL, Fouts ET, Midthun K, Newburg DS. Human milk mucin inhibits rotavirus replication and prevents experimental gastroenteritis. J Clin Invest. 1992;90:1984-91.

30. Dekker J, Tytgat KM. Binding of rotavirus by a $46 \mathrm{kD}$ milk-glycoprotein may prevent gastroenteritis. J Pediatr Gastroenterol Nutr. 1993;17:228-30.

31. Gaur P, Munjhal A, Lal SK. Influenza virus and cell signaling pathways. Med Sci Monit. 2011;17(6):RA148-54.

32. Lv S, Dai C, Liu Y, et al. Cell surface protein C23 affects EGF-EGFR induced activation of ERK and PI3K-AKT pathways. J Mol Neurosci. 2015;55:519-24. https://doi.org/10.1007/s12031-014-0375-7.

33. Dent P. Crosstalk between ERK, AKT, and cell survival. Cancer Biol Ther. 2014; 15(3):245-6. https://doi.org/10.4161/cbt.27541.

34. Martinez FD. Heterogeneity of the association between lower respiratory illness in infancy and subsequent asthma. Proc Am Thorac Soc. 2005;2:15761. https://doi.org/10.1513/pats.200504-044AW.

35. Martinez FD. The origins of asthma and chronic obstructive pulmonary disease in early life. Proc Am Thorac Soc. 2009;6(3):272-7. https://doi.org/10. 1513/pats.200808-092RM

36. Leigh $R$, Proud D. Virus-induced modulation of lower airway diseases: pathogenesis and pharmacologic approaches to treatment. Pharmacol Ther. 2015;148:185-98. https://doi.org/10.1016/j.pharmthera.2014.12.005.

37. Sichelstiel A, Yadava K, Trompette A, et al. Targeting IL-1B and IL-17A driven inflammation during influenza-induced exacerbations of chronic lung inflammation. PLoS One. 2014;9(2):e98440. https://doi.org/10.1371/ journal.pone.0098440.

38. Zhang C, Lee HJ, Shrivastava A, et al. Long-term in vitro expansion of epithelial stem cells enabled by pharmacological inhibition of PAK1-ROCKmyosin II and TGF- $\beta$ signaling. Cell Rep. 2018;25(3):598-610. https://doi.org/ 10.1016/j.celrep.2018.09.072.

\section{Publisher's Note}

Springer Nature remains neutral with regard to jurisdictional claims in published maps and institutional affiliations.

Ready to submit your research? Choose BMC and benefit from:

- fast, convenient online submission

- thorough peer review by experienced researchers in your field

- rapid publication on acceptance

- support for research data, including large and complex data types

- gold Open Access which fosters wider collaboration and increased citations

- maximum visibility for your research: over $100 \mathrm{M}$ website views per year

At $\mathrm{BMC}$, research is always in progress.

Learn more biomedcentral.com/submissions 